ARTÍCULO

\title{
Autorregulación de las interacciones escritoras: una aproximación metodológica con universitarios
}

\author{
Mauricio Ortega González' \\ Felipe de Jesús Patrón Espinosal (D \\ Naneli López Alvarado' (D) \\ Virginia Pacheco Chávez" (D)
}

\section{RESUMEN}

Una de interacciones escritoras más complejas es cuando un escritor revisa y corrige un texto elaborado por él mismo, o sea, una interacción escritora autorregulada. Una posibilidad metodológica para su estudio es cuando un escritor tiene contacto directo con aquello de lo que escribe y es él mismo quien funge como su propio lector para la corrección de su escrito. En este estudio se evaluaron los efectos del contacto directo de escritores con el referente sobre las modificaciones de las descripciones elaboradas. Veinte participantes escritores, describieron por escrito la ruta para ir de un lugar a otro, recorrieron dicha ruta descrita y corrigieron sus propios textos en diferentes momentos. Después, ochenta lectores recorriendo la ruta descrita por los escritores para evaluar la precisión de los textos. Los resultados muestran que quienes modificaron sus textos después de recorrer la ruta reelaboraron sus escritos de forma más precisa, pero no necesariamente más congruente.

PALABRAS CLAVE

autorregulación; escritura; lectura; revisión; universitarios. 
SELF-REGULATION OF WRITING INTERACTIONS: A

\title{
METHODOLOGICAL APPROACH WITH COLLEGE STUDENTS
}

\begin{abstract}
One of the most complex writing interactions is when writers review and modify a text prepared by themselves, that is, a self-regulated writing interaction. A methodological possibility for the study of these interactions is when writers have direct contact with what they are writing, and it is they who serve as their own reader for the correction of their writing. In this study, the effects of direct contact of writers with the referent on the modifications of the descriptions made were evaluated. Twenty participating writers described in writing the route to go from one place to another, traveled the described route, and corrected their own texts at different times. Then, eighty readers traveled the route described by the writers to assess the accuracy of the texts. The results show that those who modified their texts after traveling the route rewrote their texts more precisely, but not more congruently.
\end{abstract}

KEYWORDS

self-regulation; writing; reading; revision; college students.

\section{AUTORREGULAÇÃO DE INTERAÇÕES DE ESCRITA: UMA ABORDAGEM METODOLÓGICA COM ESTUDANTES UNIVERSITÁRIOS}

\section{RESUMO}

Uma das interações de escrita mais complexas é quando um escritor revisa e corrige um texto preparado por ele mesmo, ou seja, uma interação de escrita autorregulada. Uma possibilidade metodológica para o estudo desse tipo de interação é quando um escritor tem contato direto com o que ele escreve e é ele mesmo quem atua como o seu próprio leitor para a correção de sua escrita. Neste estudo foram avaliados os efeitos do contato direto dos autores com o referente nas modificações das descrições elaboradas. Vinte escritores participantes descreveram por escrito a rota para ir de um lugar para outro, percorreram a rota descrita e corrigiram seus próprios textos em momentos diferentes. Depois, oitenta leitores percorreram o caminho descrito pelos escritores para avaliar a precisão dos textos. Os resultados mostram que aqueles que modificaram seus textos depois de percorrer o percurso refizeram seus escritos com mais precisão, mas não necessariamente com mais congruência.

PALABRAS CLAVE

autorregulação; escrita; leitura; revisão; estudantes universitários. 


\section{INTRODUCCIÓN}

Uno de los principales intereses de la psicología, en lo que refiere al estudio del lenguaje escrito, gira en torno a las interacciones escritoras como promotoras del comportamiento complejo, es decir, su papel funcional en el aprendizaje y la generación de conocimiento (Irigoyen, Acuña y Jiménez, 2017; Langer y Applebee, 1987; Ortega González, 2015). Desde la psicología se han elaborado diversos modelos para explicar el desarrollo de la conducta de escribir, particularmente de los paradigmas cognitivo y psicolingüístico, con importantes aportes a nivel teórico y metodológico en la enseñanza educativa de la lengua escrita (Alvarado y Silvestri, 2003; Hayes y Flower, 1980; MacArthur y Graham, 2015; Saddler, 2012; Zimmerman, 2008; Zimmerman y Risemberg, 1997).

Gran parte de investigación en el área de la composición de textos ha sido desarrollada bajo los modelos de Flower y Hayes (1981) y de Bereiter y Scardamalia (1987), quienes han reportado que los escritores expertos se distinguen de los novatos en que los primeros son capaces de elaborar sus escritos teniendo en cuenta a la audiencia a la que se dirigen, pueden hablar sobre lo que van a escribir por anticipado, revisan continuamente su texto para verificar si se ajusta a los objetivos que se plantearon inicialmente $y$, con base en ello, corrigen o modifican sus escritos sin necesidad de la participación de alguien más que valide dicha correspondencia (Alvarado y Silvestri, 2003; Carlino 2005; Castelló, Bañales Faz y Vega López, 2010; Castelló, Iñesta y Monereo, 2009; Pacheco Chávez, 2010).

Estas últimas características, revisar y corregir lo que se está escribiendo, son críticas en las interacciones de un escritor durante la elaboración de su texto. Al respecto, existe evidencia empírica que muestra que los escritores expertos pueden cumplir simultáneamente las funciones típicas del lector anticipando las reacciones de este ante el texto. El escritor experto es capaz de regular su propio comportamiento sin necesidad de interactuar con alguien más, es decir, se trata de una interacción escritora autorregulada (Bangert-Drown, Hurley y Wilkinson, 2004; Ortega González, 2015; Ortega González, Pacheco Chávez y Carpio Ramírez, 2014; Pacheco Chávez, 2010; Pacheco Chávez, Ortega González y Carpio Ramírez, 2013).

Este planteamiento coincide con lo dicho por Zimmerman y Kitsantas (2002) quienes desde una perspectiva cognitiva social sostienen que el desarrollo de las habilidades escritoras comienza cuando un individuo es regulado socialmente para después alcanzar la autorregulación en la composición de escritos. Por ejemplo, los docentes durante la enseñanza o instrucción de la lengua escrita tienen por objetivo explicitar los errores en los escritos de sus alumnos en aspectos ortográficos, sintaxis, semántica, lógica de uso de expresiones particulares, entre otros. Solo hasta un momento posterior, con base en varios ejercicios, los alumnos son capaces de independizarse de las fuentes de corrección del profesor y son ellos mismos quienes se autocorrigen (Goldstein, 2004; Graham, Harris y Santagelo, 2015).

Estos autores, Zimmerman y Kitsantas (2002), más que clasificar a los escritores en expertos y novatos, destacan que los escritores pueden adquirir diferentes niveles de habilidades en forma secuencial y progresiva: observación, emulación, autocontrol y autorregulación. La principal influencia para la transición entre estos 
niveles es el aspecto motivacional del escritor que depende de su percepción o creencia de autoeficacia. Así pues, desde esta postura, la autorregulación concibe a una persona que escribe capaz de adaptar su desempeño a las condiciones contextuales y personales para hacer uso de diferentes estrategias de ajuste con determinados resultados esperados.

Con base en las propuestas mencionadas se han sugerido dos formas generales de estudio para la autorregulación de las interacciones escritoras. La primera de ellas es mediante el análisis de las modificaciones que hace un escritor a su propio texto con base en las conductas del lector, es decir, a partir del tipo de actividad que proporciona un lector (p.ej. retroalimentación) el escritor está en condiciones de reelaborar su texto (Sato y Matsushima, 2006).

La segunda forma consiste en el uso de diferentes técnicas introspectivas, como el protocolo de análisis verbal, para la recolección de datos durante la ejecución de una tarea escrita en la que el escritor verbaliza en voz alta su pensamiento antes, durante o después del momento en que escribe (Alonso-Arroyo et al., 2016; Requena, 2003). La finalidad de estos protocolos en voz alta cuando se usan en tareas de escritura es aproximarse al orden en que se desarrollan los procesos de razonamiento y reflexión del escritor durante la elaboración de su texto. Así, estos informes verbales permiten indagar cuál es la relación funcional que existe entre el comportamiento observable y el proceso del pensamiento (Ericsson y Simon, 1980; Shimoff, 1986). A continuación, se detallan ambas aproximaciones metodológicas.

\section{ANÁLISIS DE LAS CONDUCTAS DEL LECTOR}

La investigación en el comportamiento de escribir se ha centrado en el análisis de diversos parámetros involucrados como la historia interactiva referencial del escritor en relación a lo que va a escribir (Pacheco Chávez y Villa Soto, 2005), las habilidades y competencias escritoras (Mares, 1988), el lector o audiencia potencial a la que se dirige el texto (Gunel, Hand y Dermott, 2009), la complejidad del tema de aquello que se escribe (Pacheco Chávez, Ortega González y Carpio Ramírez, 2013), las características conductuales del lector que tiene contacto funcional con el texto del escritor (Irigoyen, Jiménez y Acuña, 2006), entre otros.

Sin embargo, el parámetro más estudiado se relaciona con el análisis de los efectos que produce el comportamiento del lector en el proceso elaboración de textos del escritor, es decir, cómo la conducta del lector es capaz de modular la manera en que un escritor produce sus escritos. Esto es así porque una de las tantas funciones del lector es retroalimentar el comportamiento del escritor en diferentes tipos y niveles de complejidad (Hattie y Timperley, 2007; Panadero y Alonso-Tapia, 2014; Sato y Matsushima, 2006). La retroalimentación es entendida de forma general como aquellas respuestas de tipo convencional que proporciona un lector y que modulan la conducta del escritor (Kepner, 1991; Sato y Matsushima, 2006; Zamel, 1985; Ziv, 1984).

$\mathrm{Al}$ respecto, se han documentado distintos efectos en función del tipo de respuestas que proporciona el lector. Algunas de ellas han consistido en símbolos en los textos del escritor (Hillocks, 1982; Hyland, 2003), en comentarios específicos 
y generales de aspectos formales (p.ej. ortografía, uso de conectores y formato del texto) hasta de congruencia del texto (p.ej. orden de ideas e hilo conductor entre párrafos (Dorow y Boyle, 1998) y la modalidad de la retroalimentación (comentarios orales o escritos - Sperling, 1996).

Ahora bien, gran parte de las investigaciones coinciden en señalar que cuando un escritor tiene contacto con las diversas formas de comportamiento del lector (p.ej.comentarios al texto) hay mayor probabilidad de revise y modifique con mayor precisión quien elabora el texto (Chaudron, 1984; López et al., 2020; Pacheco Chávez, Ortega González y Carpio Ramírez, 2013; Sato y Matsushima, 2006). No solo eso, la mayoría de las veces las respuestas de los lectores pueden influir de un modo tan decisivo en el comportamiento del escritor, que incluso puede llegar a motivar a que oste sustituya sus escritos por otros completamente distintos. Además, esto se puede extender a situaciones de aprendizaje de una segunda lengua mediante la composición de escritos (Farris, 2002; Gascoigne, 2004).

$\mathrm{Al}$ respecto, Bitchener, Young y Cameron (2005) evaluaron los efectos del tipo de retroalimentación en la precisión y en los errores lingüísticos en la elaboración de textos de estudiantes de aprendizaje de lenguaje inglesa. Formaron tres grupos de retroalimentación de tipo correctiva durante diferentes semanas $(2,4$, 8 y 12) en un curso: el primer grupo con corrección en el escrito y conferencia, el segundo grupo únicamente corrección en el escrito y el tercer grupo sin corrección. La corrección general de los dos primeros grupos consistió en la explicitación de errores lingüísticos como las preposiciones, oraciones en pasado simple, definición de artículos, gerundios, tiempo de los verbos, etc. Mientras que la conferencia, que solo tuvo el primer grupo consistía en preguntar al profesor y recibir información adicional a la explicación y los ejemplos. Al término del curso se les pidió a los estudiantes que escribieran una carta en inglés a un conocido que estuviera en el extranjero. En la carta debían de responder a una serie de preguntas que su conocido extranjero deseaba saber acerca de ellos y su familia (p.ej. en dónde vive, cuántos hermanos, qué actividades ha realizado). Los resultados muestran que el grupo con retroalimentación y conferencia fue más preciso en la composición de sus textos en distintos niveles (preposiciones, pasado simple, artículos definidos) que los otros dos grupos en el periodo de 12 semanas.

Es necesario resaltar dos aspectos importantes de los estudios experimentales antes mencionados. El primer aspecto es que la mayoría de estos estudios hacen énfasis en el análisis de los textos y dejan de lado las condiciones que constituyen propiamente la interacción escritora, como la complejidad del tema en términos del número de relaciones implicadas que referir (p.ej. describir la apariencia física de un objeto o describir la ruta para de llegar de un lugar a otro en una ciudad), la historia interactiva referencial del lector con dicho tema (p.ej. familiaridad o dominio del tema), el modo en que entra en contacto con el escrito (p.ej. directo o indirecto), etc. El segundo aspecto por mencionar es que las categorías de análisis utilizadas en los textos son del tipo formal (ortográfico, gramatical y sintáctico) más que del tipo conductual interactivo, por ejemplo, de precisión, congruencia y coherencia en términos de ajuste y de mediación del comportamiento de otro o uno mismo. 


\section{ANÁLISIS DEL PROTOCOLO VERBAL}

Una alternativa metodológica para el estudio de la autorregulación del comportamiento escritor es el informe verbal. El análisis de protocolo de pensamiento en voz alta es el más común de estos informes que tiene el propósito conocer cómo funcionan las distintas operaciones cognitivas que ordenan y construyen las ideas que son plasmadas en textos, es decir, se pretende analizar el proceso del pensamiento del escritor en el momento que elabora su texto (Cepeda, 2016; Ericsson y Simon, 1980; Plancarte et al., 2013; Shimoff, 1986).

Una reciente investigación acerca de la autorregulación en el comportamiento escritor mediante el empleo del análisis de protocolo verbal fue el realizado por Rincón Camacho, Sanabria Rodríguez y López Vargas (2016), quienes se propusieron identificar las conductas específicas que participan en la formulación de un modelo de procesamiento cognitivo y autorregulatorio en la escritura universitaria. Les pidieron a estudiantes universitarios elaborar un texto científico a partir de un fragmento de un texto que los investigadores les proporcionaron. La tarea consistió en elaborar por escrito los párrafos anterior y posterior de dicho fragmento mientras simultáneamente verbalizaban sus pensamientos. Después de elaborar los textos se les realizó una entrevista a los participantes analizar las verbalizaciones de los pensamientos posteriores respecto a los escritos. Los resultados mostraron que los participantes utilizaron con más frecuencia estrategias de revisión a nivel local (ortografía, gramática y puntuación), que de planificación en la que generalmente se enfocaron en los niveles básicos de procesamiento como la búsqueda de secuencia de segmentos encadenados para formar frases.

\section{PLANTEAMIENTO DEL PROBLEMA}

Las dos aproximaciones metodológicas descritas adolecen de varios problemas de tipo conceptual y metodológico. Respecto a la primera aproximación, existe la gran dificultad de estudiar las interacciones escritoras autorregulatorias ya que siempre se depende de las respuestas del lector a quien escribe, pues invariablemente están sujetas a un tiempo indefinido posterior a la elaboración del escrito. Esto último, no es un problema metodológico de la temporalidad de su evaluación o un problema inherente a la relación misma. Más bien, la concepción misma interacción escritora es ser valorada por un lector. El escritor siempre escribe en función del lector, incluso cuando escribe para sí mismo, pues simultáneamente funge como un lector de aquello escribe, por ejemplo, en ocasiones escribimos para nosotros mismos con el propósito de aclarar una idea. En este sentido, el escritor es al mismo tiempo el primer lector del texto que elabora.

A lo anterior hay que agregar que con frecuencia se analiza de manera independiente de las características del texto que se elabora, de las habilidades de quien escribe, de los momentos en que el escritor corrige su texto y de la situación general en que se desarrolla la interacción escritora. 
Por otro lado, el protocolo verbal posee varios inconvenientes para el estudio de las interacciones escritoras, ya sean estas autorreguladas o no (Cabello y O'Hora, 2002). El principal problema radica en la difícil verificación de correspondencia entre el lenguaje oral y el lenguaje escrito al momento de elaborar un texto. Es cierto que ambas formas de lenguaje (oral y escrito) poseen grandes similitudes y una continuidad funcional una de la otra (Kroll,1981), no obstante, son diferentes en sus desarrollos cuyas particularidades sintácticas, gramaticales y lexicales que las rigen no siempre se corresponden (Ong, 1982; Vygotsky, 1988, 1979).

Una dificultad adicional de los protocolos verbales que impacta directamente en los aspectos metodológicos es que se sustentan en la falsa concepción dualista cartesiana del hombre compuesto por dos sustancias cualitativamente distintas, influenciada por el mecanicismo de la física renacentista (Ryle, 2002). La visión dualista del comportamiento de escribir sostiene que aquello que se escribe es consecuencia directa de lo que previamente se puede decir en forma oral, y que este último a su vez es resultado de diversos procesos cognitivos (o cerebrales como sostienen algunos modernos) previos al hablar. Al respecto, Ferreiro (2003) menciona que esta postura fue predominante entre los lingüistas, pues se creía que la actividad escritora era una representación deformada del lenguaje oral. Se asumía que existía una primacía o un dominio del nivel fonológico por encima de alguno otro y que la escritura era un producto solo y únicamente para representar de forma correcta todo lo que ocurre en un nivel fonológico.

Ahora bien, es necesario mencionar que, independientemente del tipo de metodología, existe una confusión que pocos en el área parecen haberse percatado: el término autorregulación se usa de manera indiscriminada como un concepto teórico y a la vez metodológico. Si bien existe una estrecha relación entre una teoría científica y su correspondiente metodología (véase esta problemática en Carnap, 1989), también es cierto que son distintos niveles de lenguaje. Es decir, el lenguaje teórico y el lenguaje metodológico necesariamente están vinculados, pero no de forma directa ya que los conceptos teóricos son abstractos, mientras que los metodológicos tiene lugar como referentes concretos en tiempo y espacio físico que posibilita su análisis experimental. El problema surge cuando se le da el estatus de variable independiente o dependiente a un término que no tiene un referente concreto en particular. Esto genera la ilusión de que se está estudiando la autorregulación al incluir el término en los objetivos experimentales. Claramente se trata de un error del tipo categorial (Ryle, 2002).

Una alternativa teórica, y por tanto metodológica, para el estudio de las interacciones escritoras autorreguladas puede derivarse del modelo de campo de la psicología interconductual propuesta por Kantor $(1975,1978)$. Con base en este modelo, la interacción escritora autorregulada estaría ubicada en un marco general comportamiento psicológico y no como una teoría particular y aislada acerca de 
la conducta de escribir. ${ }^{1}$ La interacción escritora, desde la postura interconductual kantoriana, se conforma por tres elementos relacionados entre sí: referidor (escritor), referido (lector) y referente (aquello de lo que se escribe). Las características de referidor y referido van desde las habilidades y competencias escritoras y lectoras, hasta la familiaridad que tienen con el referente (historia interactiva referencial) para establecer la interacción referencial (Figura 1).

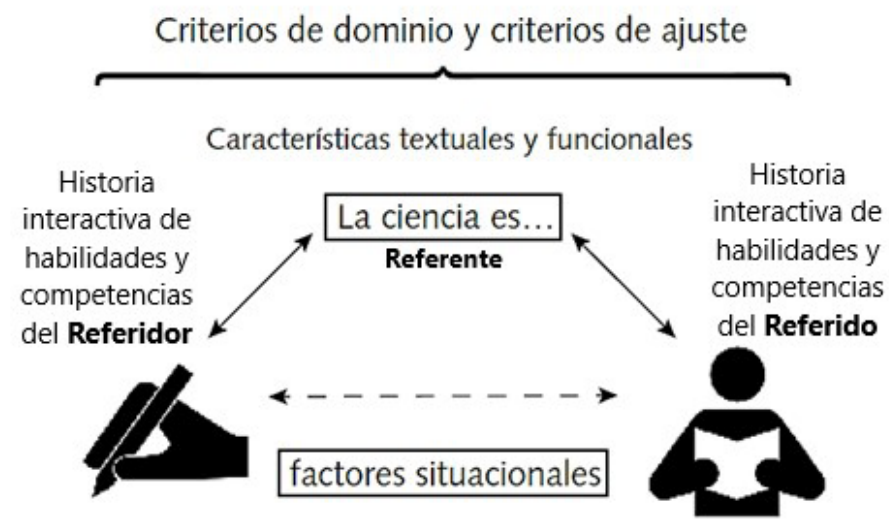

Figura 1 - Representación de interacción escritora (Tomado de Pacheco Chávez, Ortega González y Carpio Ramírez, 2013).

Las diferentes formas de interacción que se pueden concebir a partir de la organización de esos tres elementos amplían la gama de posibilidades metodológicas para el estudio del comportamiento escritor, incluyendo el autorregulado. En este sentido, una posibilidad de la autorregulación del comportamiento escrito pudiera ocurrir cuando el escritor (referidor) tiene contacto directo con aquello de lo que escribe (referente), y así propiciar la revisión y corrección del texto que elabora él mismo (referido).

Por ejemplo, cuando damos indicaciones por escrito de cómo llegar de un punto $A$ a un punto $B$ y nosotros mismos verificamos la correspondencia de nuestro escrito. Una situación como esta no es inusual en la vida cotidiana, pue si bien es raro que una persona escriba una indicación para seguirla él mismo, también es cierto que escribimos y verificamos para recordarlo posteriormente como ayuda o sustituto de la memoria. Esta verificación, que puede darse en diferentes momentos (antes, durante y después de realizar un escrito), amplía las diferentes formas de revisión y corrección de un texto a partir del contacto funcional con aquello que refiere de forma escrita. Una tarea como la mencionada posibilita la actividad diferencial de quien elabora un texto, lo cual tiene

1 A lo largo de este texto seguiremos usando la noción de interacción escritora únicamente con fines de expositivos para facilitar la compresión del estudio. 
importantes implicaciones funcionales, en aspectos de precisión y congruencia en la composición de textos.

El presente estudio tuvo por objetivo evaluar los efectos del contacto directo de escritores con la ruta descrita por ellos mismos en diferentes etapas, sobre la precisión y congruencia en la elaboración de textos.

\section{MÉTODO}

\section{PARTICIPANTES}

Participaron 100 universitarios (64 mujeres y 36 hombres) cuyo rango de edad oscilaba entre los 19 y los 25 años $(\bar{X}=22,83 \pm 3,25)$. Del total de participantes, 60 eran pertenecientes a la Facultad de Ciencias Humanas de la Universidad Autónoma de Baja California, por tanto, estaban familiarizados con los espacios e instalaciones de la facultad. Los 40 participantes restantes no tenían ninguna familiaridad o contacto previo con las instalaciones, es decir, no tenían una historia interactiva referencial con las instalaciones de la facultad. Para corroborar esto se realizó una breve entrevista a cada uno de ellos. La elección de población universitaria para el estudio obedece a las habilidades y competencias mínimas de escritura y lectura que estos han desarrollado para alcanzar dicho nivel académico, esto quiere decir que cumplen con las condiciones necesarias interactivas en la elaboración de textos académicos. Se les informó a todos que se les daría un incentivo económico por su colaboración, esto con el propósito de motivar a los participantes para un mejor desempeño en el estudio.

\section{SITUACIÓN EXPERIMENTAL}

El experimento se llevó a cabo dentro de las instalaciones dentro de la Facultad de Ciencias Humanas. El estudio inició en el Laboratorio para la Investigación del Comportamiento (LICOM) y finalizó en diferentes áreas de la facultad.

\section{MATERIALES}

Se emplearon hojas blancas tamaño carta y bolígrafos de diferente color para la elaboración y modificación de los textos.

\section{PROCEDIMIENTO}

Antes de iniciar las sesiones experimentales se explicó a todos los participantes que la investigación tenía el propósito de conocer la forma en que nos comunicamos con otras personas. Se decidió por expresarles a los participantes este propósito con la intención de establecer las condiciones típicas y cotidianas en las que se escribe, así como de evitar contaminar las variables de interés del estudio. El estudio consistió en dos sesiones generales; una sesión para los escritores y una sesión para los lectores, tal y como se describe continuación.

\section{Sesión para los escritores}

Del total de participantes familiarizados con las instalaciones de la facultad se seleccionaron de forma aleatoria a 20 de ellos. Estos fungieron como escritores 
y fueron conducidos uno por uno al LICOM. Ahí se les dijo que la tarea a realizar consistía en describir por escrito una ruta para llegar de un lado a otro dentro de la facultad (Figura 2). A continuación, se detalla cómo se llevó a cabo esta sesión para los escritores la cual estuvo conformada por tres distintas etapas.

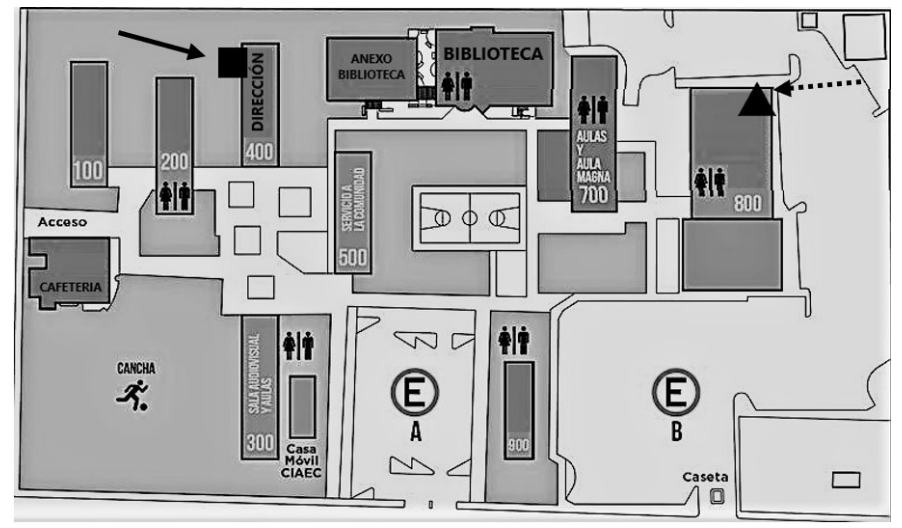

Figura 2 - Plano de la Facultad de Ciencias Humanas. La flecha punteada señala el punto inicial (LICOM) representado por un triángulo negro, mientras que la flecha uniforme señala el punto final (Dirección) representado por un cuadrado negro. La distancia promedio entre los dos puntos era de 240 metros, pues esta dependía de la ruta que cada participante describió.

Fuente: Base de datos de investigación.

Elaboración de los autores.

\section{Etapa 1 - Elaboración de descripción escrita de la ruta}

Se le proporcionó a cada participante una hoja con instrucciones por escrito y un espacio en blanco para escribir. Se le pidió que leyera en voz alta las instrucciones:

¡Hola! Agradecemos tu participación en nuestro estudio ya que nos ayudará a conocer la forma en la que desarrollamos diversas habilidades de comunicación. Tu tarea consiste en describir por escrito cómo llegar de aquí, el Laboratorio para la Investigación del Comportamiento, a la Dirección de la Facultad. Debes de ser específico en tu descripción, ya que alguien más lo leerá y realizará el recorrido que escribas. Si tienes alguna duda pregúntale al investigador.

\section{Etapa 2 - Recorrido de la ruta}

Después de que los participantes elaboraron sus escritos se les pidió que leyeran en voz alta sus descripciones de la ruta al mismo tiempo que realizaban el recorrido de esta, es decir, cada participante siguió su propia ruta para verificar la correspondencia entre la ruta y su texto. Los investigadores acompañaron en todo momento a los escritores en su recorrido verificando que los participantes siguieran sus propias instrucciones. 
Etapa 3 - Modificación de la descripción escrita con base en el recorrido realizado

Todos los escritores fueron asignados al azar a uno de los cuatro grupos conformados por 5 participantes cada uno, como a continuación se describe. Se les instigó a modificar sus textos en distintos momentos en función del grupo:

- Grupo Modificación Previa (MPre). A los participantes de este grupo se les pidió revisar sus textos y hacer las modificaciones pertinentes a los escritos antes de realizar el recorrido de la ruta, pues después ya no podrían alterar su escrito.

- Grupo Modificación Durante (MD). A los participantes de este grupo se les pidió revisar sus textos y hacer las modificaciones pertinentes a los escritos durante el recorrido de la ruta.

- Grupo Modificación Posterior (MPos). A los participantes de este grupo se les pidió revisar sus textos y hacer las modificaciones pertinentes a su texto después del recorrido de la ruta.

- Grupo Modificación Sin Previo Aviso (MSPA). A los participantes de este grupo se les pidió revisar sus textos y hacer las modificaciones pertinentes a los escritos antes de realizar el recorrido de la ruta, igual que el grupo MPre, con la excepción de que después del recorrido se les dijo sin previo aviso que podían volver a realizar modificaciones a su texto si así lo consideraban.

La sesión de cada participante terminó después de realizar el recorrido y de entregar las modificaciones de sus escritos, en caso de haberlo hecho. Es necesario señalar que todos los participantes utilizaron bolígrafos de distinto color para la elaboración de los textos (color negro) y para las modificaciones sobre sus propios textos (color rojo). Esto permitió a los investigadores distinguir entre los textos que escribieron inicialmente (originales) de aquellos que fueron corregidos (modificados).

Los investigadores procedieron a reproducir copias fotostáticas de todos los escritos ocultando las partes modificadas, de tal manera que fue posible obtener 40 textos en total: 20 textos originales y 20 textos modificados.

\section{Sesión para los lectores}

Los 80 participantes restantes del estudio fungieron como lectores y fueron conducidos uno por uno al LICOM. Ahí se le proporcionó un escrito original o uno modificado de forma aleatoria a cada uno de los lectores. Se les explicó que el escrito describía una ruta para llegar a un lugar específico dentro de la facultad y que debían leerlo para llegar al punto indicado.

Del total de lectores, 40 estaban familiarizados con las instalaciones de la facultad, o sea, disponían de una historia interactiva referencial (CHR) con el referente (instalaciones de la facultad). Los 40 lectores restantes no disponían de una historia interactiva referencial (SHR), es decir, no estaban familiarizados. Con base en esta característica de historia interactiva referencial de los lectores, se procedió a proporcionar los textos originales y modificados de los diferentes grupos de 
escritores como se muestra en la Tabla 1. La inclusión de los participantes lectores tuvo el propósito servir de indicador para determinar el índice de precisión de los textos originales y los modificados, y así contrastar el papel que juega la exposición de la ruta en la interacción escritora.

Tabla 1 - Asignación de textos originales y modificados a los lectores.

\begin{tabular}{|c|c|c|c|c|}
\hline $\begin{array}{c}\text { Textos de } \\
\text { grupo }\end{array}$ & \multicolumn{2}{|c|}{$\begin{array}{r}\text { Lectores Con Historia } \\
\text { Referencial (CHR) }\end{array}$} & \multicolumn{2}{c|}{$\begin{array}{c}\text { Lectores Sin Historia } \\
\text { Referencial } \\
\text { (SHR) }\end{array}$} \\
\hline & Texto Original & Texto Modificado & Texto Original & Texto Modificado \\
\hline MPre & 5 participantes & 5 participantes & 5 participantes & 5 participantes \\
\hline MD & 5 participantes & 5 participantes & 5 participantes & 5 participantes \\
\hline MPos & 5 participantes & 5 participantes & 5 participantes & 5 participantes \\
\hline MSPA & 5 participantes & 5 participantes & 5 participantes & 5 participantes \\
\hline
\end{tabular}

MPre: Grupo Modificación Previa; MD: Grupo Modificación Durante; MPos: Grupo Modificación Posterior; MSPA: Grupo Modificación Sin Previo Aviso. Fuente: Base de datos de investigación. Elaboración de los autores.

La sesión de los lectores terminaba cuando estos indicaron el punto final de acuerdo con el texto. En la Figura 3 se muestra el diseño general del estudio y la distribución general de las sesiones de los participantes escritores y lectores

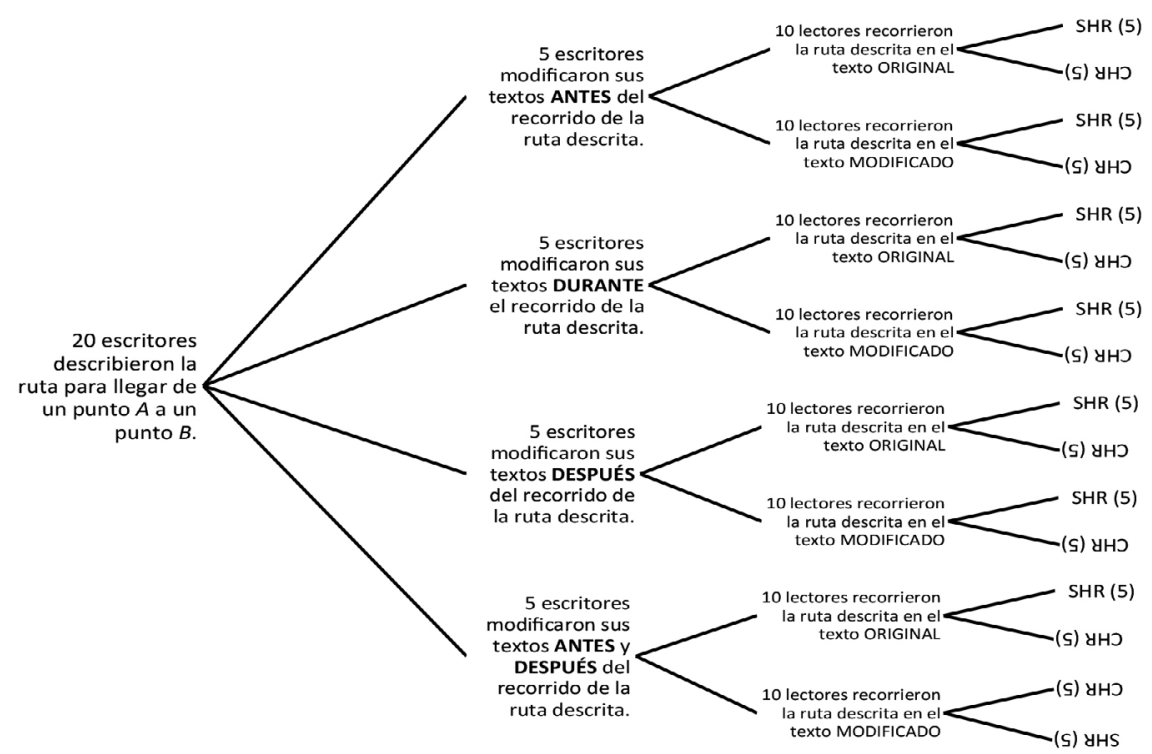

Figura 3 - Diseño general del estudio experimental.

CHR: Lectores Con Historia Referencial; SHR: Lectores Sin Historia Referencial.

Fuente: Base de datos de investigación.

Elaboración de los autores. 


\section{ANÁLISIS DE DATOS}

Se efectuó un análisis de datos intrasujeto de los escritores con el propósito de obtener representatividad funcional, es decir, un análisis individual en términos cuantitativos. Las categorías y medidas para el análisis de datos se describen a continuación.

Se estableció que debían evaluarse los textos de los escritores con base en dos categorías generales: precisión y congruencia. Se consideró que en la categoría de precisión los textos de tipo instruccional deberían incluir los elementos necesarios y suficientes que permitieran a una persona, distinta de quien escribió el texto, realizar el recorrido de un lugar a otro. Se eligió como indicador las ejecuciones de los lectores en términos espaciales de aproximación al punto final. Para ello se procedió a dividir a la facultad por zonas mediante líneas imaginarias con diferentes valores y así evaluar el desempeño de los lectores CHR y SHR. En la Figura 4 se muestra el plano general de la facultad dividido en cuadrantes distribuidos de forma proporcional con sus respectivos valores cuya asignación se realizó con base en dos criterios: la proximidad y lejanía entre el punto inicial y de punto final, y las rutas posibles o únicas para transitar dentro de las instalaciones de la facultad. Así, los cuadrantes más cercanos al punto final en las rutas posibles poseen un mayor valor que aquellos que se encuentran más lejanos.

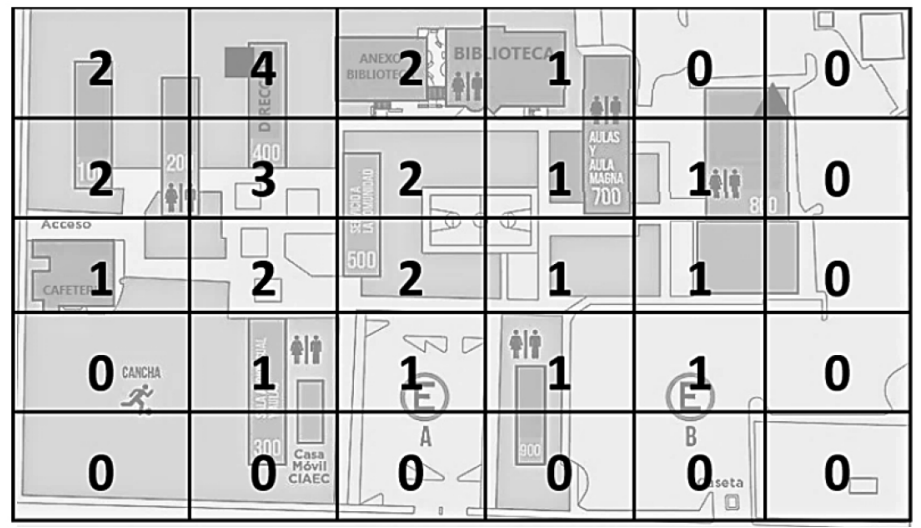

Figura 4 - Plano de la Facultad de Ciencias Humanas dividido en cuadrantes.

Fuente: Base de datos de investigación.

Elaboración de los autores.

Por otro lado, la categoría de congruencia fue definida como la correspondencia entre las referencias escritas de la ruta (p.ej. números de pasos, distancias, edificios específicos, objetos en el camino, número de giros) con las referencias concretas de la ruta. Por ejemplo, si los participantes describían en su texto "a 10 metros se encuentra una cancha de básquetbol de lado derecho", la correspondencia consistiría en la correcta especificación en distancia, objeto y ubicación de la cancha 
de básquetbol. De lo contrario, se consideraba como falta de correspondencia. Se consideraron cuatro niveles de congruencia que diferían entre sí por la referencia del lugar y la secuencia correcta, tal como se muestra en la Tabla 2.

Tabla 2 - Baremo parar analizar la congruencia de los textos elaborados por los participantes.

\begin{tabular}{|l|c|}
\hline \multicolumn{1}{|c|}{ Categoría de congruencia } & Puntos \\
\hline $\begin{array}{l}\text { Muy congruente (correspondencia de todas las referencias mencionadas). } \\
\text { Únicamente frases que describan correctamente la ubicación del inicio del } \\
\text { recorrido, del término del recorrido y de los puntos intermedios de referencia y } \\
\text { una secuencia adecuada. }\end{array}$ & 3 \\
\hline $\begin{array}{l}\text { Congruente (correspondencia en el mayor número de referencia mencionadas). } \\
\text { Al menos dos frases que describan correctamente la ubicación del inicio del } \\
\text { recorrido, del término del recorrido y de los puntos intermedios de referencia y una } \\
\text { secuencia adecuada. }\end{array}$ & 2 \\
\hline $\begin{array}{l}\text { Poco congruente (correspondencia en el menor número de referencias mencionadas). } \\
\text { Frases que describan incorrectamente la ubicación del inicio del recorrido, del término } \\
\text { del recorrido o de los puntos intermedios de referencia, pero en una secuencia adecuada. }\end{array}$ & 1 \\
\hline $\begin{array}{l}\text { Incongruente (sin correspondencia de las referencias mencionadas). } \\
\text { Únicamente frases que describan incorrectamente la ubicación del inicio del } \\
\text { recorrido, del término del recorrido o de los puntos intermedios de referencia en una } \\
\text { secuencia inadecuada. }\end{array}$ & 0 \\
\hline
\end{tabular}

Fuente: Base de datos de investigación.

Elaboración de los autores.

Con base en estos niveles de congruencia, las descripciones de los escritores fueron valoradas por tres jueces de forma independiente, apoyados en una descripción general realizada por los propios investigadores (véase Anexo 1). Se acordó un mínimo de acuerdos del 90\% para considerarse confiable. En caso de existir acuerdos por debajo de lo establecido se volvía a analizar para decidir si hay acuerdo o se anula la participación del estudiante.

\section{RESULTADOS}

En la Figura 5 se muestran cuatro planos que especifican el lugar preciso al que llegó cada uno de los lectores (L) con base en los textos. El propósito de esta figura es representar espacialmente en el desempeño individual de cada uno de los lectores.

En el primer plano (superior-izquierdo) se indica el lugar al que llegaron los lectores CHR con textos originales. Se observa que únicamente dos lectores con textos de los grupos MPre (L1) y MPos (L18) llegaron al punto final. Esto corresponde al 10\% de los lectores del grupo. El resto de los lectores no lograron llegar al lugar esperado.

En el segundo plano (superior-derecho) se muestra el lugar al que llegaron los lectores CHR con textos modificados. Lo primero que se puede apreciar es que 
TEXTOS ORIGINALES

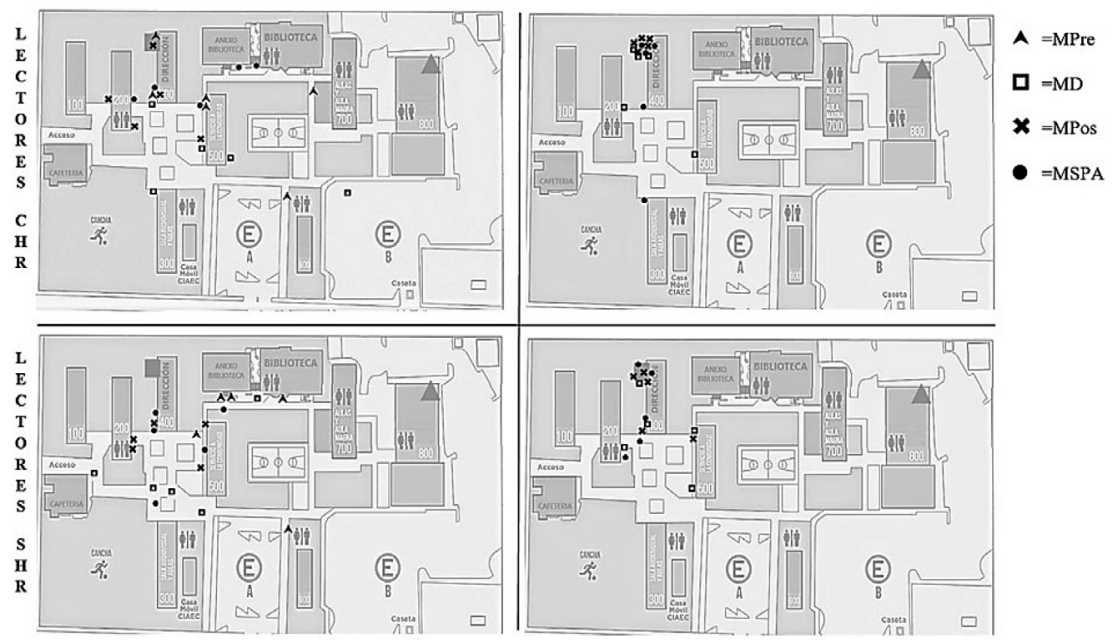

Figura 5 - Distribución espacial de la ejecución de los lectores CHR y SHR con base en los textos originales y modificados elaborados por los escritores.

MPre: Grupo Modificación Previa; MD: Grupo Modificación Durante; MPos: Grupo Modificación Posterior; MSPA: Grupo Modificación Sin Previo Aviso.

Fuente: Base de datos de investigación.

Elaboración de los autores.

no aparecen lectores con textos modificados del grupo MPre ya que ningún escritor realizó modificaciones. Teniendo en cuenta esto, se observa que once lectores se agrupan en el lugar final, entre ellos se encuentran todos los lectores pertenecientes al grupo MPos.

En el tercero plano (inferior-izquierdo) se indica el lugar al que llegaron los lectores SHR con textos originales. Se observa que ninguno de los lectores llegó al punto esperado. Los lectores más cercanos al lugar final fueron tres participantes de los grupos MSPA (L65, L67) y MD (L53).

En el cuarto plano (inferior-derecho) se muestra el lugar al que llegaron los lectores SHR con texto modificados. Se aprecia que seis lectores de los grupos MD (L42), MPos (L52, L54, L60) y MSPA (L66, L68) llegaron al punto final, y que en conjunto representan el $40 \%$ del total de lectores correspondientes a esta condición.

De manera general, al observar los cuatro planos se aprecia que los lectores $\mathrm{CHR}$ con textos modificados son quienes se encuentran más cercanos al punto final, mientras que los lectores SHR con texto original son los más lejanos.

En la Figura 6 se muestran los puntos alcanzados por los lectores con base en sus ejecuciones con textos originales y modificados. Si bien corresponden a los mismos datos que se observan en la Figura 5, aquí están representados de forma tradicional en gráficas de barras con fines de representación sistemática. 


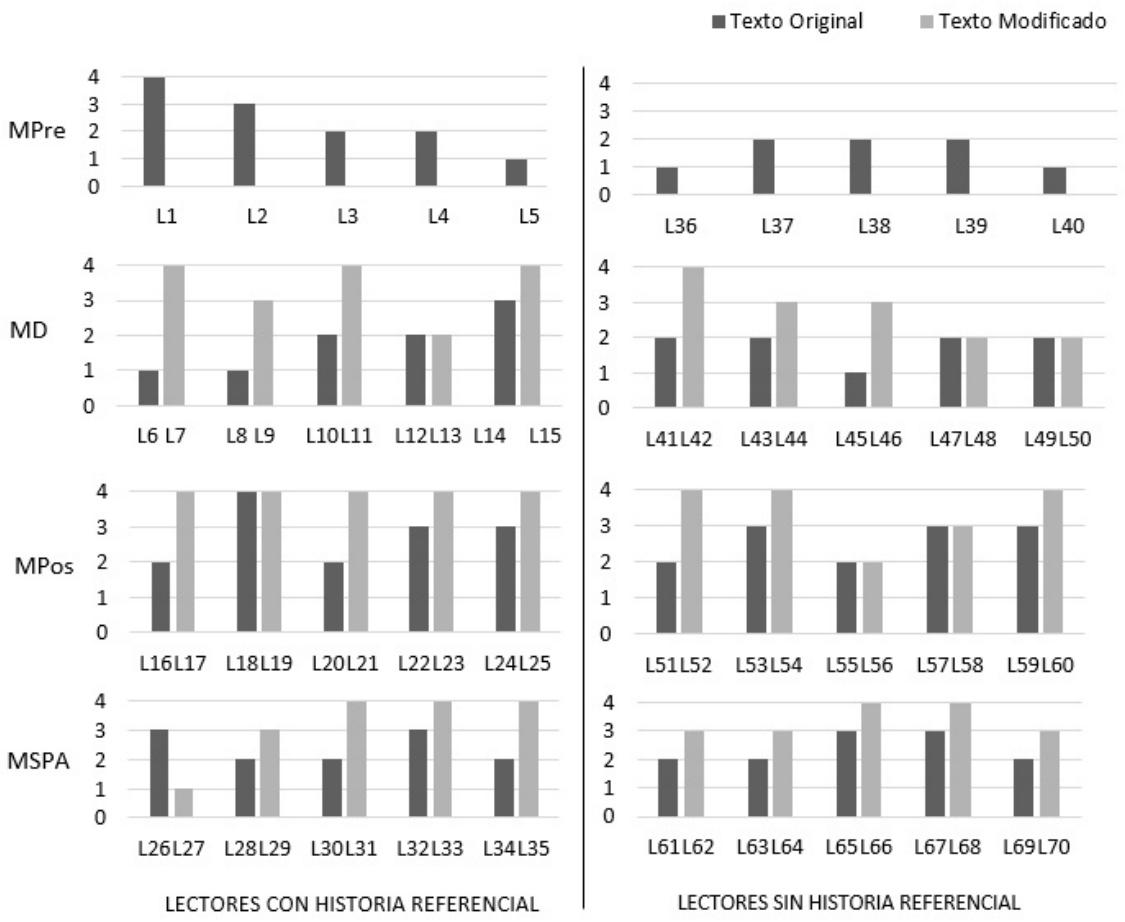

Figura 6 - Puntuación de precisión alcanzada por los lectores.

MPre: Grupo Modificación Previa; MD: Grupo Modificación Durante; MPos: Grupo Modificación Posterior; MSPA: Grupo Modificación Sin Previo Aviso.

Fuente: Base de datos de investigación.

Elaboración de los autores.

En las gráficas superiores se observa que los lectores CHR y SHR del grupo MPre con textos originales obtuvieron puntos similares. Solo un lector CHR (L1) alcanzó la máxima puntuación posible. Dado que los escritores de este grupo MPre no modificaron sus textos, no se muestra puntuación alguna.

Por otro lado, más abajo se observa que los lectores CHR y SHR del grupo $\mathrm{MD}$ con textos originales tuvieron un desempeño similar ( $\forall=2$ pts.) y que ninguno llegó al punto final. No obstante, el desempeño de los lectores con textos modificados de este grupo fue distinto; 3 lectores CHR (L7, L11, L15 lo que representa el 60\% del grupo) y un lector SHR (L42) llegaron al punto final.

Debajo de la gráfica anterior se aprecia que de los lectores CHR y SHR con textos originales del grupo MPos, solamente uno llegó al punto final (L18). En cambio, de los lectores con texto modificados fueron cinco CHR (L17, L19, L21, L23, L25) y tres SHR (L52, L54, L60) quienes sí llegaron a la ubicación final.

Por último, ningún lector del grupo MSPA con texto original llegó al punto final. Sin embargo, cinco lectores de este mismo grupo, pero con textos modificados llegaron al punto final; tres CHR (L31, L33, L35) y dos SHR (L66, L68). 
De manera general, se observa que la ejecución de los lectores, tanto CHR como SRH, es mejor en la condición de textos modificados que con textos originales.

En la Figura 7 se muestra el índice de congruencia alcanzado en los textos originales y modificados de los escritores. En la gráfica superior se observa que cada uno de los textos originales de los escritores del grupo MPre fue valorado como "Congruente". Debajo de esta gráfica se aprecian resultados similares en el grupo $\mathrm{MD}$, con excepción de un participante (P10) quien en su texto modificado alcanzó el índice de "Muy Congruente".

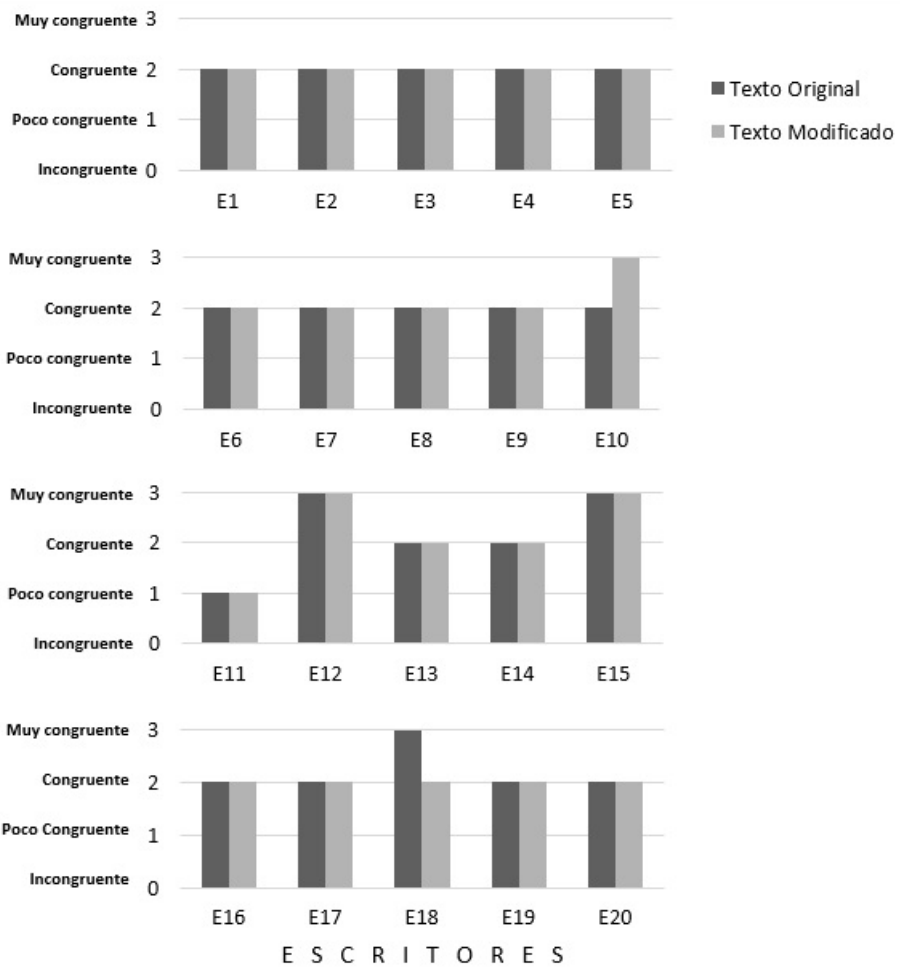

Figura 7 - Índice de congruencia de textos originales y modificados.

Fuente: Base de datos de investigación.

Elaboración de los autores.

Por otro lado, se observa que el índice de congruencia de los textos del grupo MPos no varió en sus versiones originales y modificadas. Los textos de los escritores E12 y E15 se mantuvieron como "Muy Congruente", los E13 y E14 como "Congruente" y el E11 como "Poco Congruente".

Por último, están los escritos del grupo MSPA cuyos participantes se les instigaron a modificar sus textos antes de hacer el recorrido. Hasta ese momento se encontraban en las mismas condiciones que los escritores del grupo MPre, y 
de quienes su desempeño fue igual que dicho grupo pues no realizaron ninguna modificación. Sin embargo, después de realizar el recorrido se les dijo que tenían la oportunidad de modificar los textos y lo hicieron. Los textos originales y modificados de este grupo fueron valorados como "Congruente" con excepción del escrito E18 en su versión original fue "Muy Congruente".

\section{DISCUSIÓN}

Los resultados del estudio muestran de forma consistente que aquellos participantes escritores que tuvieron contacto directo con la ruta realizaron modificaciones a sus textos que favorecieron la precisión de estos. Mientras que los escritores que no tuvieron contacto directo con la ruta (referente) no modificaron y fueron poco precisos, como se observó en el grupo MPre. Este resultado coincide parcialmente con lo encontrado en la literatura respecto a los efectos de las respuestas de la audiencia o de lectores y la continua revisión del texto en el comportamiento del escritor (Pacheco Chávez, Ortega González y Carpio Ramírez, 2013; Sato y Matsushima, 2006). Es posible afirmar que existen altas probabilidades de que el comportamiento escritor autorregulado se vea alterado si existe un contacto directo con el referente, es decir, con aquello de lo que se escribe. Esto suponemos se debe a que el contacto directo con el referente en un comportamiento escritor permite mediar un mayor número de relaciones de elementos del referente que estructuren la interacción escritora que no ocurre cuando no hay contacto directo.

Consideramos que el contacto directo con el referente puede ser una condición de promoción de interacciones escritoras autorreguladas al momento de desarrollar un texto. Esto se confirma si compara la condición particular del grupo MSPA, a quienes se les instigó a modificar sus textos antes y después del recorrido. En un primer momento se encontraban en las mismas condiciones que los escritores del grupo MPre y cuyo desempeño fue similar en ambos grupos. No obstante, después de realizar el recorrido los participantes del grupo MSPA reelaboraron sus textos haciéndolos más precisos, como demuestran las ejecuciones de los lectores.

No obstante, la modificación de textos no supuso mayor congruencia, entendida esta como la correspondencia entre descripción de la ruta y la ruta propiamente. Los resultados muestran que el contacto directo no afectó la congruencia en la mayoría de los textos originales y modificados. Esto sugiere que una interacción escritora puede desarrollarse de forma precisa, pero no forzosamente una forma congruente. Cabe mencionar que una forma de comportamiento psicológico congruente en las interacciones escritoras implica un ajuste conductual en términos de la organización funcional del referente como correspondencia entre el decir y hacer, que es modulada por el tipo de referencia e historia interactiva referencial particular del propio escritor (Carpio Ramírez, 1994; Pacheco Chávez, Ortega González y Carpio Ramírez, 2013; Ribes-Iñesta, 2004).

En la parte introductoria de este escrito se mencionó que en psicología el interés principal respecto al comportamiento escritor está en la propia interacción y no en los vestigios o productos. Por el contrario, es necesario observar lo que se escribe y a quién se dirige cuando se escribe, en qué momento, bajo que situaciones físicas y sociales, qué método, en qué momento vuelve a leer y corrige, 
etc. Tomar en consideración tales variables significa inclinarse por un análisis interactivo psicológico.

Es claro que en función del tipo de texto elaborado, la situación comunicativa, los géneros discursivos, propósitos comunicativos y otras más unidades gramaticales, son pertinentes en psicología sólo en la medida en que en el análisis se utilicen métricas correspondientes a las interacciones conductuales. Justamente en este trabajo se proponen las medidas de precisión y congruencia (como correspondencias entre los elementos referido, referidor y referente) y así evitar el uso de la lógica formal clásica en la evaluación de textos (Pacheco Chávez, Ortega González y Carpio Ramírez, 2011; Varela, 1992).

Respecto a los desempeños de los participantes lectores, estos fueron los indicadores de precisión para evaluar los textos y cuyo papel funcional implica necesariamente considerar su historia interactiva referencial como parte de un análisis interactivo (Gunel, Hand y Dermott, 2009; Hayes y Flower, 1980; Pacheco Chávez, 2010). Creemos que la correspondencia entre el decir (del escritor) y el hacer (del lector) como práctica efectiva y pertinente permite el análisis de las interacciones escritoras reguladas (cuando el escritor y lector son distintas personas) y autorreguladas como práctica congruente (cuando el escritor y lector es la misma persona). Los resultados coinciden las investigaciones realizadas bajo diferentes modelos de la composición escrita (Flower y Hayes, 1981; Scardamalia y Bereiter, 1986; Zimmerman y Risemberg, 1997) en que la conducta escritora se ve afectada por la historia interactiva referencial de quien escribe y su relación con el potencial lector.

Si bien la ejecución entre lectores fue consistente con base en el texto asignado, se sugiere que para próximas investigaciones se realice una prueba de lectura que descarte posibles deficiencias en la comprensión lectora de estos participantes y, por tanto, obtener datos más exactos de las interacciones escritoras.

Por otro lado, consideramos que la tarea experimental está diseñada para propiciar la participación del escritor en la elaboración de su propio texto, ya que reúne las condiciones necesarias que permiten cumplir las funciones del escritor y lector centradas en una sola persona. Además, la preparación metodológica utilizada es más acorde para un análisis funcional interactivo de la conducta de escribir en el que es posible estudiar el papel funcional del escritor y lector.

Para próximos estudios es necesario comparar experimentalmente otras condiciones particulares del referente, por ejemplo, la complejidad de este en términos del número y tipo de relaciones implicadas. Algunas de ellas pudieran ser dimensiones de referencia a un evento futuro o pasado (tiempo) y lugar distante (espacio) respecto a la interacción presente. O bien, referencia de un evento u objeto con una dimensión de apariencia en términos del tipo de contacto funcional lingüístico abstracto (v. g. autodescribir las virtudes y defectos de quien escribe) o concreto (v.g. la autodescripción física de quien escribe). En otras palabras, se sugiere podría ser otra morfología de la escritura misma, como un ensayo o un poema, y no solo en episodios en el que se solicita el seguimiento de indicaciones que uno mismo elaboró en otro momento. Consideramos que el tipo de relación funcional con referente conforma la complejidad del comportamiento escritor regulado o autorregulado en su totalidad. 
Por otro lado, quizá debería considerarse situaciones en el que los participantes escritores vayan haciendo sus descripciones de cómo llegar al lugar al mismo tiempo que van haciendo la ruta, y ya no simplemente un contacto directo como estrategia de corrección.

Sin duda, un aspecto que queda pendiente es el correspondiente análisis cualitativo. $\mathrm{Al}$ respecto hay que decir que actualmente uno de los retos más difíciles en el estudio de las interacciones escritoras se relaciona con el desarrollo de unidades de medida propias para su análisis experimental. La mayoría de las métricas o escalas empleadas son del tipo estructural (legibilidad, redacción, estilo) y del tipo formal (números de palabras escritas, diversidad lexical, uso correcto de la puntuación, sintaxis, gramática, frases, vocabulario). Poco se ha avanzado en la creación de medidas del tipo funcional que permitan un análisis interactivo, es decir, medidas que se relacionen con la mediación del comportamiento interindividual e intraindividual en la producción de un referente escrito. No obstante, no debe pensarse en desechar los análisis formales en términos de ocurrencias o extensiones que no necesariamente forman parte de lo que exige y requiere un análisis de interacciones escritoras.

Finalmente, los resultados del presente estudio pueden contribuir al diseño de estrategias didácticas en la elaboración de textos en el contexto educativo desde diferentes aproximaciones enfocadas en la autorregulación y el aprendizaje (Rosário et al., 2016; Valle et al., 2008; Virtanen, Nevgi y Niemi, 2013). La posibilidad de elaborar textos más precisos no solo se puede ver favorecida con la revisión y corrección de escrito, sino también si se tiene contacto directo con aquello de lo que escribe y así hacer más partícipe al estudiante en la elaboración de sus textos.

\section{REFERENCIAS}

ALONSO-ARROYO, A.; FUJITA, M. S. L.; GIL-LEIVA, I.; PANDIELLA, A. Protocolo verbal: análisis de la producción científica, 1941-2013. Informação \& Sociedade: Estudos, João Pessoa, v. 26, n. 2, p. 61-76, maio/ago. 2016. Disponible en: https://brapci.inf.br/index.php/article/download/46720. Acceso en: 19 jun. 2020.

ALVARADO, M.; SILVESTRI, A. Introducción La composición escrita: procesos y enseñanza. Cultura y Educación, España, v. 15, n. 1, p. 7-15, 2003. https://doi. org/10.1174/113564003765202357

BANGERT-DROWNS, R. L.; HURLEY, M. M.; WILKINSON, B. The effects of school-based writing-to-learn interventions on academic achievement: a meta-analysis. Review of Educational Research, Chicago, v. 74, n. 1, p. 29-58, 2004. https://doi. org/10.3102/00346543074001029

BEREITER, C.; SCARDAMALIA, M. The psychology of written composition. New Jersey: Lawrence Erlbaum Associates, 1987.

BITCHENER, J.; YOUNG, S.; CAMERON, D. The effect of different types of corrective feedback on ESL student writing. Journal of Second Language Writing, United Kingdom, v. 14, n. 3, p. 191-205, 2005. https://doi.org/10.1016/j. jslw.2005.08.001 
CABELLO, F.; O'HORA, D. Addressing the limitations of protocol analysis in the study of complex human behavior. International Journal of Psychology and Psychological Therapy, España, v. 2, n. 2, p. 115-130, 2002. Disponible en: https:// www.ijpsy.com/volumen2/num2/41/addressing-the-limitations-of-protocol-analysisEN.pdf. Acceso en: 19 jun. 2020.

CARLINO, P. Escribir, leer y aprender en la universidad. México: Fondo de Cultura Económica, 2005.

CARNAP, R. El carácter metodológico de los conceptos teóricos. In: OLIVÉ, L.; PÉREZ, A. R. (comps.). Filosofía de la ciencia y observación. 2. ed. México: Fondo de Cultura Económica, 1989. p. 70-115.

CARPIO RAMÍREZ, C. Comportamiento animal y teoría de la conducta. In: HAYES, L.; RIBES, E.; LÓPEZ, F. (coords.). Psicología interconductual: contribuciones en honor a J. R. Kantor. Guadalajara: Universidad de Guadalajara, 1994. p. 45-68.

CASTELLÓ, M.; BAÑALES FAZ, G.; VEGA LÓPEZ, N. Enfoques en la investigación de la regulación de escritura académica: Estado de la cuestión. Electonic Journal of Research in Educational Psychology, Almeria, v. 8, n. 22, p. 1.253-1.282, 2010. Disponible en: https:/www.redalyc.org/pdf/2931/293122000015.pdf. Acceso en: 19 jun. 2020. https://doi.org/10.25115/ejrep.v8i22.1424

CASTELLÓ,M.;INESTA,A.;MONEREO, C.Hacia la escritura académica autorregulada: un estudio exploratorio con estudiantes posgraduados en un entorno de aprendizaje situado. Electonic Journal of Research in Educational Psychology, Almeria, v. 7, n. 19, p. 1.1071.130, 2009. Disponible en: https://www.redalyc.org/pdf/2931/293121984009.pdf. Acceso en: 19 jun. 2020. http://dx.doi.org/10.25115/ejrep.v7i19.1336

CEPEDA, M. L. Comportamiento complejo humano. Perspectivas conductuales. México: UNAM, 2016.

CHAUDRON, C. The effects of feedback on students' composition revisions. RELC Journal,Singapore,v.15,n.2,p.1-14,1984.https://doi.org/10.1177/003368828401500201 DOROW, L.; BOYLE, M. Instructor feedback for college writing assignments in introductory classes. Journal of Behavioral Education, United Kingdom, v. 8, p. 115129, 1998. https://doi.org/10.1023/A:1022820925481

ERICSSON, A.; SIMON, H. Protocol analysis: verbal reports as data. Cambridge: MIT Press, 1980.

FARRIS, D. Treatment of error in second language writing. Ann Arbor: The University Michigan Press, 2002.

FERREIRO, E. Vigencia de Jean Piaget. México: Siglo XXI, 2003.

FLOWER, L.; HAYES, J. A cognitive process theory of writing. College Composition and Communication, United States, v. 32, n. 4, p. 365-387, 1981. http://dx.doi. org $/ 10.2307 / 356600$

GASCOIGNE, C. Examining the effect of feedback in beginning L2 composition. Foreign Language Annals, Alexandria, v. 37, n. 1, p. 71-76, 2004. https://doi. org/10.1111/j.1944-9720.2004.tb02174.x 
GOLDSTEIN,L.M. Questions and answers about teacher written commentary and student revision: teachers and students working together. Journal of Second Language Writing, United Kingdom, v. 13, n. 1, p. 63-80, 2004. https://doi.org/10.1016/j.jslw.2004.04.006

GRAHAM, S.; HARRIS, K. R.; SANTAGELO, T. Research-based writing practices and the common core: meta-analysis and meta-sinthesis. The Elementary School Journal, v. 115, n. 4, p. 498-522, 2015.

GUNEL, M.; HAND, B.; MCDERMOTT, M. A. Writing for different audiences: effects on high-school students' conceptual understanding of biology. Learning and Instruction, United Kingdom, v. 19, n. 4, p. 354-367, 2009. https://doi.org/10.1016/j. learninstruc.2008.07.001

HATTIE,J.; TIMPERLEY,H.The power of feedback. Review of Educational Research, Chicago, v. 77, n. 1, p. 81-112, 2007. https://doi.org/10.3102/003465430298487

HAYES, J.; FLOWER, L. Identifying organization of writing process. In: GREGG, L. W.; STEINBERG, E. R. (eds.). Cognitive processes in writing: an interdisciplinary approach. Hillsdale, NJ: Lawrence Erlbaum Associates, 1980. p. 3-30.

HILLOCKS, G. The interaction of instruction, teacher comment, and revision in teaching the composing process. Research in the Teaching English, United States, v. 16, n. 3, p. 261-278, 1982.

HYLAND, F. Focusing on form: student engagement with teacher feedback. System, United Kingdom, v. 31, n. 2, p. 217-230, 2003. https://doi.org/10.1016/S0346251X(03)00021-6

IRIGOYEN, J. J.; ACUÑA, K. F.; JIMÉNEZ, M. Y. (coords.). Aportes conceptuales y derivaciones tecnológicas en psicología y educación. Hermosillo, México: Qartuppi, 2017. https://doi.org/10.29410/QTP.17.01

IRIGOYEN, J.J.;JIMÉNEZ, M. Y.; ACUÑA, K. F. Evaluación de modos lingüísticos en estudiantes universitarios. Enseñanza e Investigación en Psicología, v. 11, n. 1, p. 81-95, 2006.

KANTOR, J. R. Psychological linguistics. Revista Mexicana de Análisis de la Conducta, México, v. 1, n. 2, p. 249-268, 1975. http://dx.doi.org/10.5514/rmac. v1.i2.25242

KANTOR, J. R. Psicología interconductual. Un ejemplo de construcción científica sistemática. México: Trillas, 1978.

KEPNER, C. G. An experiment in the relationship of types of written feedback to the development of second-language writing skills. The Modern Language Journal, New Jersey, v. 75, n. 3, p. 305-313, 1991. https://doi.org/10.2307/328724

KROLL, B. Developmental relationships between speaking and writing. In: KROLL, B. M.; VANN, R. J. (eds.). Connections and contrasts. Urbana: National Council of Teachers of English, 1981.p. 32-54.

LANGER, J. A.; APPLEBEE, A. N. How writing shapes thinking: a study of teaching and learning. Urbana: National Council of Teachers of English, 1987. (NCTE Research Report, 22). Disponible en: https://wac.colostate.edu/docs/books/langer_applebee/ langer_applebee.pdf. Acceso en: 19 jun. 2020. 
LÓPEZ, A.; ACUÑA, K. F.; IRIGOYEN,J.J.;JIMÉNEZ, M. Habilitación lingüística en universitarios: escritura, revisión y corrección en díadas. Enseñanza e Investigación en Psicología, México, v. 2, n. 1, p. 60-72, 2020.

MACARTHUR, C.; GRAHAM, S. Writing research from a cognitive perspective. In: MACARTHUR, C. A.; GRAHAM, S.; FITZGERALD, J. (eds.). Handbook of writing research. New York: Guilford Press, 2015. p. 187-207.

MARES, G. Análisis experimental de la relación entre diferentes competencias lingüísticas. 1988. 163 f. Tesis (Maestría en Psicología) - Universidad Nacional Autónoma de México, México, 1988. Disponible en: http://132.248.9.195/ pmig2017/0085139/Index.html. Acceso en: 19 jun. 2020.

ONG, W. Oralidad y escritura. Tecnologías de la palabra. México: Editorial Fondo de Cultura Económica, 1982.

ORTEGA GONZÁLEZ, M. Efectos de consecuencias en la emergencia de autosuplementación en la elaboración de textos en universitarios. 2015. 174 f. Tesis (Doctorado en Psicología) - Universidad Nacional Autónoma de México, México, 2015. Disponible en: http://132.248.9.195/ptd2015/septiembre/0735104/Index.html. Acceso en: 19 jun. 2020.

ORTEGA GONZÁLEZ, M.; PACHECO CHÁVEZ, V.; CARPIO RAMÍREZ, C. Efectos de consecuencias diferenciales en la elaboración de textos por universitarios. Revista Electrónica de Psicología Iztacala, México, v. 17, n. 3, p. 1.254-1.281, 2014. Disponible en: http://www.iztacala.unam.mx/carreras/psicologia/psiclin/vol17num3/ Vol17No3Art16.pdf. Acceso en: 19 jun. 2020.

PACHECO CHÁVEZ, V. ¿Se enseña a escribir a los universitarios? Análisis y propuestas desde la teoría de la conducta. México: UNAM, 2010.

PACHECO CHÁVEZ, V.; ORTEGA GONZÁLEZ, M.; CARPIO RAMÍREZ, C. Apuntes en busca de una métrica psicológica: el caso de la elaboración de texto. In: PACHECO, V.; CARPIO, C. (coords.). Observación y métricas en el análisis del comportamiento humano. Estado de México: Universidad Nacional Autónoma de México, 2011.p. 105-127.

PACHECO CHÁVEZ, V.; ORTEGA GONZÁLEZ, M.; CARPIO RAMÍREZ, C. Efectos de la respuesta del lector y el uso de ejemplos sobre la composición escrita. Revista Colombiana de Psicología, Bogotá, v. 22, n. 1, p. 13-34, 2013. Disponible en: https://www.redalyc.org/pdf/804/80428081002.pdf. Acceso en: 19 jun. 2020.

PACHECO CHÁVEZ, V.; VILLA SOTO, J. C. El comportamiento del escritor y la producción de textos científicos. Revista Mexicana de Investigación Educativa, México, v. 10, n. 27, p. 1.201-1.224, 2005. Disponible en: https://www.redalyc.org/ pdf/140/14002715.pdf. Acceso en: 19 jun. 2020.

PANADERO, E.; ALONSO-TAPIA,J.¿Cómo nuestros alumnos? Revisión del modelo cíclico de Zimmerman sobre autorregulación del aprendizaje. Anales de Psicología, Murcia, v. 30, n. 2, p. 450-462, 2014. http://dx.doi.org/10.6018/analesps.30.2.167221 PLANCARTE, P.; MORENO, D.; HICKMAN, H.; ARROYO, R.; CEPEDA, M. L. Use of analysis of verbal protocols in the study of complex human behavior. In: 
GARCÍA, C.; CORRAL, V.; MORENO, D. (eds.). Recent Hispanic research on sustainable behavior and interbehavioral psychology. Hauppauge: Nova Science Publishers, 2013. p. 159- 178.

REQUENA, M. El análisis de protocolo como técnica para la comprensión de los procesos de razonamiento. Laurus, Caracas, v. 9, n. 16, p. 79-96, 2003. Disponible en: https://www.redalyc.org/pdf/761/76111331007.pdf. Acceso en: 19 jun. 2020.

RIBES-IÑESTA, E. Acerca de las funciones psicológicas: un post-scriptum. Acta Comportamentalia: Revista Latina de Análisis del Comportamento, México, v. 12, n. 2, p. 117-127, 2004.

RINCÓN CAMACHO, L.; SANABRIA RODRÍGUEZ,L. B.; LÓPEZ VARGAS, O. Aproximación a un modelo de autorregulación en escritura académica a partir del análisis de protocolos. Folios, Bogotá, n. 43, p. 59-79, 2016. http://dx.doi. org/10.17227/0123487043folios59.76

ROSÁRIO, P. S. L. F.; FUENTES, S.; BEUCHAT, M.; RAMACIOTTI, A. Autorregulación del aprendizaje en una clase de la universidad: un enfoque de infusión curricular. Revista de Investigación Educativa, Salamanca, v. 34, n. 1, p. 31-49, 2016. http://dx.doi.org/10.6018/rie.34.1.229421

RYLE, G. El concepto de lo mental. Buenos Aires: Paidós, 2002.

SADDLER, B. Teacher's guide to effective sentence writing. New York: Guilford, 2012.

SATO, K.; MATSUSHIMA, K. Effects of audience awareness on procedural text writing. Psychological Reports, United States, v. 99, n. 1, p. 51-73, 2006. http://doi. org/10.2466/pr0.99.5.51-73

SCARDAMALIA, M.; BEREITER, C. Research on written composition. In: WITTROCK, M. (ed.). Handbook of research on teaching. New York: Macmillan, 1986. p. 778-803.

SHIMOFF, E. Post-session verbal reports and the experimental analysis of behavior. The Analysis of Verbal Behavior, United States, v. 4, p. 19-22, 1986. http://dx.doi. org/10.1007/BF03392811

SPERLING, M. Revisiting the writing-speaking connection: challenges for research on writing and writing instruction. Review of Educational Research, Chicago, v. 66, n. 1, p. 53-86, 1996. http://dx.doi.org/10.2307/1170726

VALLE, A.; NÚÑEZ, J. C.; CABANACH, R. G.; GONZÁLEZ-PINEDA, J. A.; RODRÍGUEZ, S.; ROSÁRIO, P.; CEREZO, R.; MUÑOZ-CADAVID, M. A. Self-regulated profiles and academic achievement. Psicothema, España, v. 20, n. 4, p. 724-731, 2008.

VARELA, J. Cuando se escribe algo de lo que se ha leído: gramática o conducta lingǘstica. Revista Mexicana de Análisis de la Conducta, v. 18, n. 1-2, p. 83-102, 1992. VIRTANEN, P.; NEVGI, A.; NIEMI, H. Self-regulation in higher education: students' motivational, regulational an learning strategies, and their relationships to study success. Studies for the Learning Society, Denmark, v. 3, n. 1-2, p. 20-36, 2013. http://dx.doi. org/10.2478/sls-2013-0004 
VYGOTSKY, L. E1 desarrollo de los procesos psicológicos superiores. Barcelona: Crítica, 1979.

VYGOTSKY, L. Pensamiento y lenguaje. México: Quinto Sol, 1988.

ZAMEL, V. Responding to student writing. TESOL Quarterly, Alexandria, v. 19, n. 1, p. 79-101, 1985. http://dx.doi.org/10.2307/3586773

ZIMMERMAN, B. J. Investigating self-regulation and motivation: historical background, methodological developments, and future prospects. American Educational Research Journal, United States, v. 45, n. 1, p. 166-183, 2008. https:// doi.org/10.3102/0002831207312909

ZIMMERMAN, B.J.; KITSANTAS, A. Acquiring writing revision and self-regulatory skill through observation and emulation. Journal of Educational Psychology, Washington, v. 94, n. 4, p. 660-668,2002.http://dx.doi.org/10.1037/0022-0663.94.4.660 ZIMMERMAN, B.; RISEMBERG, R. Becoming a self-regulated writer: a social cognitive perspective. Contemporary Educational Psychology, United States, v. 22, n. 1, p. 73-101, 1997. https://doi.org/10.1006/ceps.1997.0919

ZIV, N. The effect of teacher comments on the writing of four college freshmen. In: BEACH, R.; BRIDWELL, L. S. (eds.). New directions in composition research. New York: The Guilford Press, 1984. p. 362-380.

\section{SOBRE LOS AUTORES}

Mauricio Ortega González es doctor en psicología por la Universidad Nacional Autónoma de México (México). Profesor de la Universidad Autónoma de Baja California (México).

E-mail: mauricio.ortega40@uabc.edu.mx

Felipe de Jesús Patrón Espinosa es doctor en ciencia del comportamiento (ciencias biológicas y agropecuarias) por la Universidad de Guadalajara (México). Profesor de la Universidad Autónoma de Baja California (México).

E-mail: felipe.patron@uabc.edu.mx

Naneli López Alvarado es estudiante graduada en psicología por la Universidad Autónoma de Baja California (México).

E-mail: naneli.lopez@uabc.edu.mx

Virginia Pacheco Chávez es doctora en pedagogía por la Universidad Nacional Autónoma de México (México). Profesora de la misma institución. E-mail: vpacheco@unam.mx 


\section{ANEXO 1}

\section{DESCRIPCIÓN GENERAL DE LA RUTA ELABORADA POR LOS INVESTIGADORES PARA EL ANÁLISIS DE LOS ESCRITOS}

Te encuentras en el Laboratorio para la Investigación del Comportamiento (LICOM). Ahora debes caminar por el pasillo hasta topar con la pared. Mientras caminas por pasillo verás a unos 5 metros de tu lado izquierdo el Laboratorio de Psicofisiología y de tu lado derecho el Laboratorio del Desarrollo del Potencial Humano.

Cruzas otra puerta de vidrio, continúas caminando hasta tener de tu lado derecho el salón 804, ahí veras las escaleras que deberás bajar y te llevarán a la planta baja. Ya estando ahí, deberás caminar derecho pasando una reja de fierro, continua derecho hasta la cancha de básquetbol. Sube el escalón de la cancha y continúa caminando hasta que encuentres de tu lado derecho un camino de concreto, tomarás ese camino y quedarás mirando justo frente a biblioteca, pasarás la escultura de piedra de tu lado izquierdo y una vez que suceda doblarás a la izquierda.

Sigue caminando derecho hasta pasar una pared con anuncios, del edificio 500 de tu lado izquierdo. Una vez que lo pases doblas ligeramente a la izquierda e inmediatamente a la derecha (tendrás el huerto de tu lado derecho), pasas la pared del edificio 400 que estará de tu lado derecho e inmediatamente giras a la derecha. Camina a lo largo del pasillo hasta llegar la última puerta y habrás llegado a dirección. 